\title{
Evaluación del comportamiento de un biofertilizante en el cultivo de chile habanero (Capsicum chinense JACQ.) Variedad Jaguar®
}

Evaluation of the behavior of a biofertilizer in the cultivation of habanero pepper (Capsicum chinense JACQ.) Jaguar® variety

Rojas Avelizapa Luz Irene, Dávila Lezama María del Rosario, Angel Lara Maria Alva, Navarro Rodríguez Ana María del Pilar, Regalado Infante Paul Edgardo

Facultad De Ciencias Biológicas y Agropecuarias Universidad Veracruzana Campus Orizaba-Córdoba

${ }^{凶}$ Autor para correspondencia: 1uzrojas@uv.mx

Recibido: 21/07/2020

Aceptado: $15 / 10 / 2020$

\section{RESUMEN}

El chile habanero (Capsicum chinense Jacq.) Solanacea semi-perenne con denominación de origen el estado de Yucatán, el cultivo es considerado con potencial debido a su alta rentabilidad, retornos económicos, competencia y demanda en el mercado de chile, este cultivo demanda una buena nutrición tanto de micronutrientes como macronutrientes, sin embargo un desconocimiento en cuanto al uso de los fertilizantes químicos ha ocasionado un incremento en el uso de estos, ocasionando daños irreversibles al medio ambiente, por lo anterior se ha implementado el uso de abonos orgánicos con el fin de recuperar la micro fauna del suelo y así estos organismos aceleren los procesos microbianos, de tal forma que se aumente las cantidades de nutrientes que pueden ser asimilados por las plantas. Por tal motivo se realizó una evaluación de un biofertilizante aplicado sobre lombricomposta en el cultivo de chile habanero variedad Jaguar y el efecto que tiene sobre el desarrollo y rendimiento bajo condiciones de invernadero en la FCBA ubicada en Peñuela municipio de Amatlán de Los Reyes Veracruz. El trasplante se realizó al mes de edad el día 23 de diciembre del 2019, se trabajó con 8 tratamientos y 1 testigo absoluto. El diseño experimental fue boques al azar, la parcela útil fue de 2 plantas y 3 repeticiones dando un total de 6 plantas evaluadas por tratamiento, dejando fuera las plantas de los extremos y las centrales. Las variables evaluadas fueron: altura de la planta, número de frutos por planta, dimensiones del fruto (largo y diámetro) y el peso unitario del fruto. Las variables se analizaron empleando el Statistical Analysis System (S.A.S). Las variables tuvieron diferencias significativas entre tratamientos. El biofertilizante que tuvo mejor efecto fue el de lombricomposta sin tamizar.

Palabras clave: Producción, Habanero, Lombricomposta, Consorcio.

\begin{abstract}
The habanero pepper (Capsicum chinense Jacq.) Solanacea semi-perennial with a denomination of origin in the state of Yucatán, the crop is considered with potential due to its high profitability, economic returns, competition and demand in the Chilean market, this crop demands a good nutrition of both
\end{abstract}


Rojas et al., 2020 micronutrients and macronutrients, however a lack of knowledge regarding the use of chemical fertilizers has caused an increase in the use of these, causing irreversible damage to the environment, therefore the use of organic fertilizers has been implemented with the In order to recover the micro fauna of the soil and thus these organisms accelerate the microbial processes, in such a way that the amounts of nutrients that can be assimilated by the plants are increased. For this reason, an evaluation of a biofertilizer applied on vermicompost in the Jaguar variety habanero pepper crop and the effect it has on the development and yield under greenhouse conditions was carried out at the FCBA located in Peñuela municipality of Amatlán de Los Reyes Veracruz. The transplant was carried out at one month of age on December 23, 2019, with 8 treatments and 1 absolute control. The experimental design was random forests, the useful plot was 2 plants and 3 repetitions giving a total of 6 plants evaluated per treatment, leaving out the plants at the ends and the central ones. The variables evaluated were: plant height, number of fruits per plant, fruit dimensions (length and diameter) and the unit weight of the fruit. The variables were analyzed using the Statistical Analysis System (S.A.S). The variables had significant differences between treatments. The biofertilizer that had the best effect was vermicompost without sieving.

Keywords: Production, Habanero, Vermicompost, Consortium.

\section{INTRODUCCIÓN}

Capsicum ssp, es una hortaliza de gran importancia alimenticia, en México se producen cuatro (C. chinense Jacq., C. frutescens L., C. annuum L., y $C$. pubescens Ruíz) de las cinco más producidas en todo el mundo (Hernández et al., 2009). México es el primer exportador de chile verde a nivel mundial y el sexto de chile seco; nuestros principales clientes Estados Unidos, Japón, Canadá, Reino Unido y Alemania. Además de un producto con presencia mundial, éste es un cultivo originario de nuestro país y parte simbólica del imaginario culinario y cultural. En 2009 destacaron Chihuahua, Sinaloa y Zacatecas como principales productores del cultivo con más la mitad del volumen nacional en su conjunto. Cabe mencionar que en el caso de Sinaloa, un estado con alto grado de tecnificación, se registró una cosecha de 40 toneladas por hectárea, en Chihuahua, 20 toneladas por hectárea, mientras Zacatecas, el de mayor superficie sembrada reportó 7 toneladas por hectárea (SIAP, 2013).
El chile habanero Capsicum Chinense tiene la característica de ser uno de los más picantes. Aunque su nombre científico alude su origen a China, en realidad es nativo del continente americano, específicamente de México y el Caribe (SIAP, 2014). El chile habanero es el chile más producido en invernadero debido a su alta rentabilidad, retornos económicos, competencia y demanda en el mercado con un precio que oscila en los 30 pesos por Kilogramo (ASERCA, 2014). El 80\% de la producción de chile habanero se comercializa como fruto fresco y el $20 \%$ restante se dirige a la elaboración de salsas, pastas y deshidratados. Se exporta principalmente a Estados Unidos, Japón, Corea del Sur, Italia y Alemania (SAGARPA, 2012).

La producción mundial de chile ha tenido un desarrollo muy alto en los últimos años, aproximadamente un $43 \%$ de incremento en superficie y un $98 \%$ de aumento en los volúmenes de producción. México ocupa el segundo lugar en volumen de producción mundial con 1, 853,610 toneladas y el tercero en 
Rojas et al., 2020

superficie cosechada con 140,693 ha, colocándose con el $8 \%$ del área y el $7 \%$ de la producción mundial en toneladas y un rendimiento promedio bajo de 13.17 ton/ha debido principalmente a la mediana y/o baja tecnología de producción que tienen varias regiones del país, le siguen Turquía, estados Unidos, España e Indonesia, representando juntos el $25 \%$ del volumen mundial de producción.

El rendimiento más alto en la producción de chile es de los productores que emplean tecnologías de alta precisión como la aplicación de riegos, fertilizantes (Vega, 2013). Sin embargo, actualmente el aumento del uso de los fertilizantes químicos en las hortalizas ha generado un incremento en los costos de producción, daño al medio ambiente y a la salud animal y humana, por lo que se está optado por alternativas de abonos orgánicos, tales como biofertilizantes, que son biopreparados que contienen células vivas o latentes de cepas microbianas eficientes de nitrógeno, solubilizadores de fosforo, potencializadoras de diversos nutrientes o productoras de sustancias activas, que se utilizan para aplicar a las semillas o al suelo, con el objeto de incrementar el número de organismos presentes en el suelo y acelerar los procesos microbianos, de tal forma que se aumente las cantidades de nutrientes que pueden ser asimilados por las plantas (Chávez, 2010).

Teniendo en cuenta lo anterior se evaluará el comportamiento del biofertilizante Consorcio, aplicado sobre lombricomposta en el cultivo del chile habanero (Capsicum chinense Jacq), variedad Jaguar, bajo condiciones de invernadero con sistema de riego por goteo con el fin de evaluar su comportamiento en cuatro variables de la producción.

\section{MATERIALES Y MÉTODOS}

La investigación se realizó durante el periodo de Enero a Mayo del 2019, en la Facultad de Ciencias Biológicas y Agropecuarias (FACBA), Campus Peñuela de la Universidad Veracruzana, Amatlán de los Reyes, Ver, región CórdobaOrizaba. Las condiciones climáticas, son templadas, su temperatura media anual oscila entre los 20 y 24 grados. La precipitación pluvial anual se encuentra entre los 1200 y $1500 \mathrm{~mm}$. El clima según las estaciones es: en verano con días calurosos y noches frescas, y los inviernos generalmente secos, no excesivos rigurosos. 


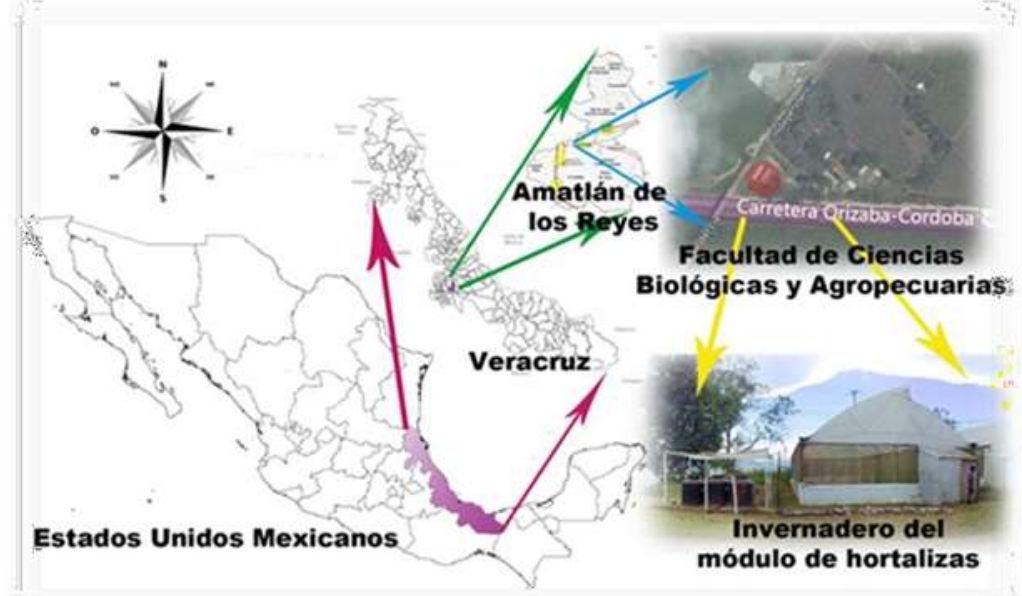

Figura 1. Ubicación del área de estudio.

El cultivo de chile habanero se estableció en un invernadero de dos aguas en forma de arco con un cenital en la parte superior. Se colocó un sistema de riego por goteo con una cintilla en el centro de la cama y un acolchado plástico. Una vez teniendo todo, el 24 de enero del 2019 se realizó el trasplante de las plántulas que tenían un mes de haber sido germinadas, se trasplantaron una distancia de 2 metros entre surcos, a una distancia de 30 centímetros entre planta y planta. Se realizaron podas cada 15 días después del trasplante y se hizo el tutorado en forma de cajón con postes. La biofertilización Consorcio se realizó por primera vez el día 28 de enero del 2019 con la ayuda de una aspersora manual.

Tabla 1. Variables evaluadas, instrumento de medición, unidad de medida y fecha de medición a las plantas de chile habanero variedad Jaguar (Capsicum chinense JACQ).

Variable evaluada Instrumento de medición / UDM

Altura de la planta

Frutos por planta

Longitud del fruto

Diámetro del fruto

Rendimiento
Cinta métrica / cm

Conteo manual / Número de frutos

Vernier / cm

Vernier / cm

Báscula/ Ton
Fecha de medición

Cada quince días a partir del día 15 de febrero de 2019

Cosecha

Cosecha

Cosecha

Cosecha 
Tabla 2. Descripción de los tratamientos aplicados al cultivo de Chile habanero variedad Jaguar (Capsicum chinense JACQ).

Tratamiento

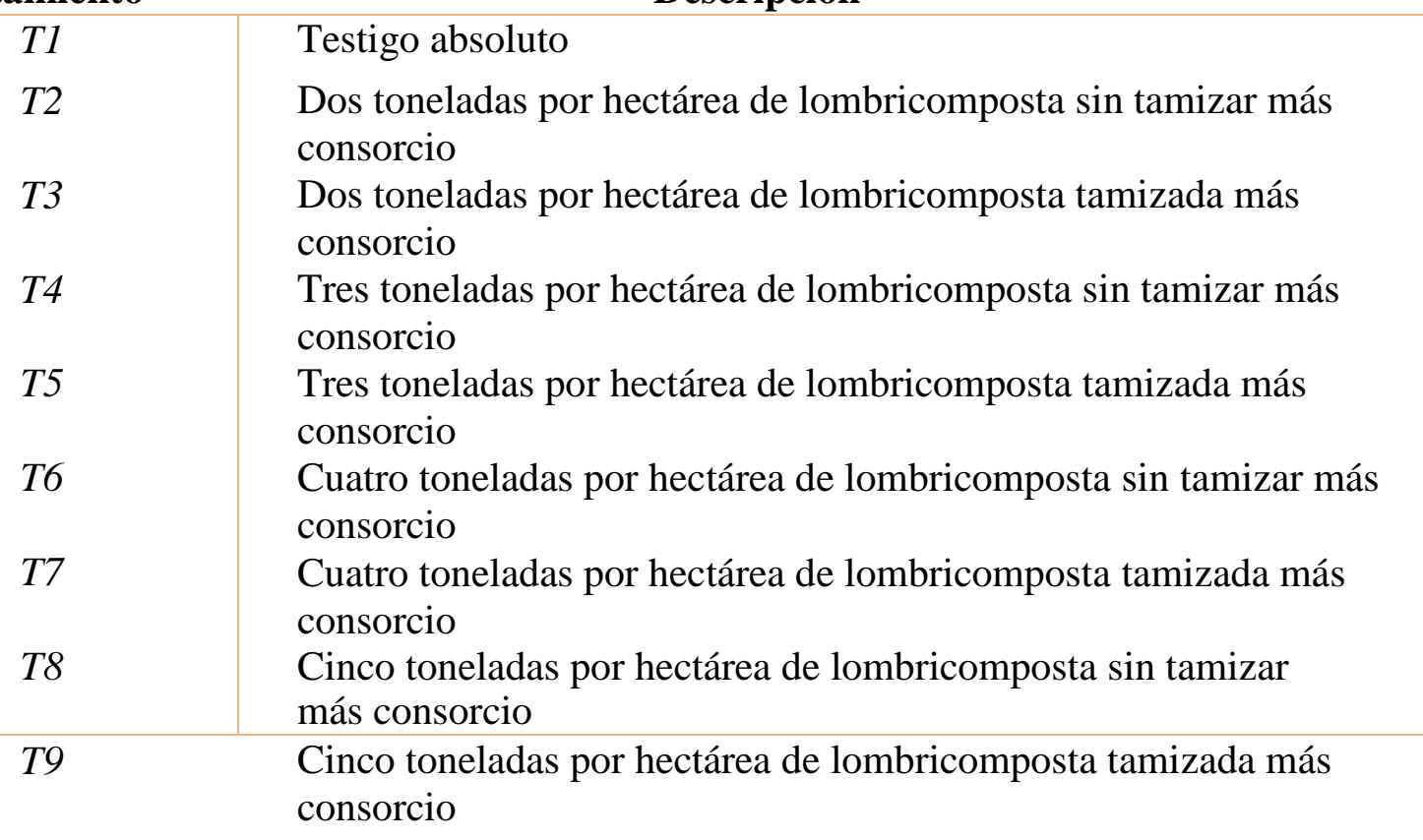

El diseño experimental fue en bloques al azar tomando como referencia el trabajo de pulido (2016), se evaluaron nueve tratamientos en total, por cada tratamiento hubo tres repeticiones. Se contó también con una parcela experimental de seis plantas por repetición y una parcela útil que evaluó la planta dos y cinco de cada tratamiento con sus respectivas repeticiones.

\section{RESULTADOS Y DISCUSIÓN}

Cabe aclarar a que debido a las condiciones climáticas todo el experimento fue concentrado en dos plantas de cada uno de los tratamientos y solo se pudieron realizar cuatro cortes, ya que se presentaron plagas y enfermedades que no permitieron el desarrollo de la planta, además de una sequía en la comunidad de Peñuela municipio de Amatlán de los Reyes, Ver., la cual fue muy estresante para ellas y hubo aborto de flores y frutos. Los resultados fueron sometidos a un análisis de varianza (ANOVA) y posteriormente se realizó una prueba de Duncan, ambos análisis se realizaron con el programa SAS.

\begin{abstract}
Altura
Se encontró diferencia significativa entre tratamientos $(\mathrm{P}=0.0235)$ según el coeficiente de variación $20 \%$ la información de este experimento se ubica en la categoría de buena. De acuerdo a la agrupación por medio de la prueba de Duncan se obtuvo que estadísticamente el tratamiento número dos fue superior al tratamiento tres y tratamiento uno, pero resultando ser igual a los demás tratamientos. Se observa que los tratamientos de lombricomposta granulada y tamizada, muestran una altura de planta de $25.85 \mathrm{~cm}$ como mínimo y $36.56 \mathrm{~cm}$ como máximo, cabe indicar que estos resultados fueron determinados al cuarto corte ya que el cultivo fue atacado por plagas.
\end{abstract}




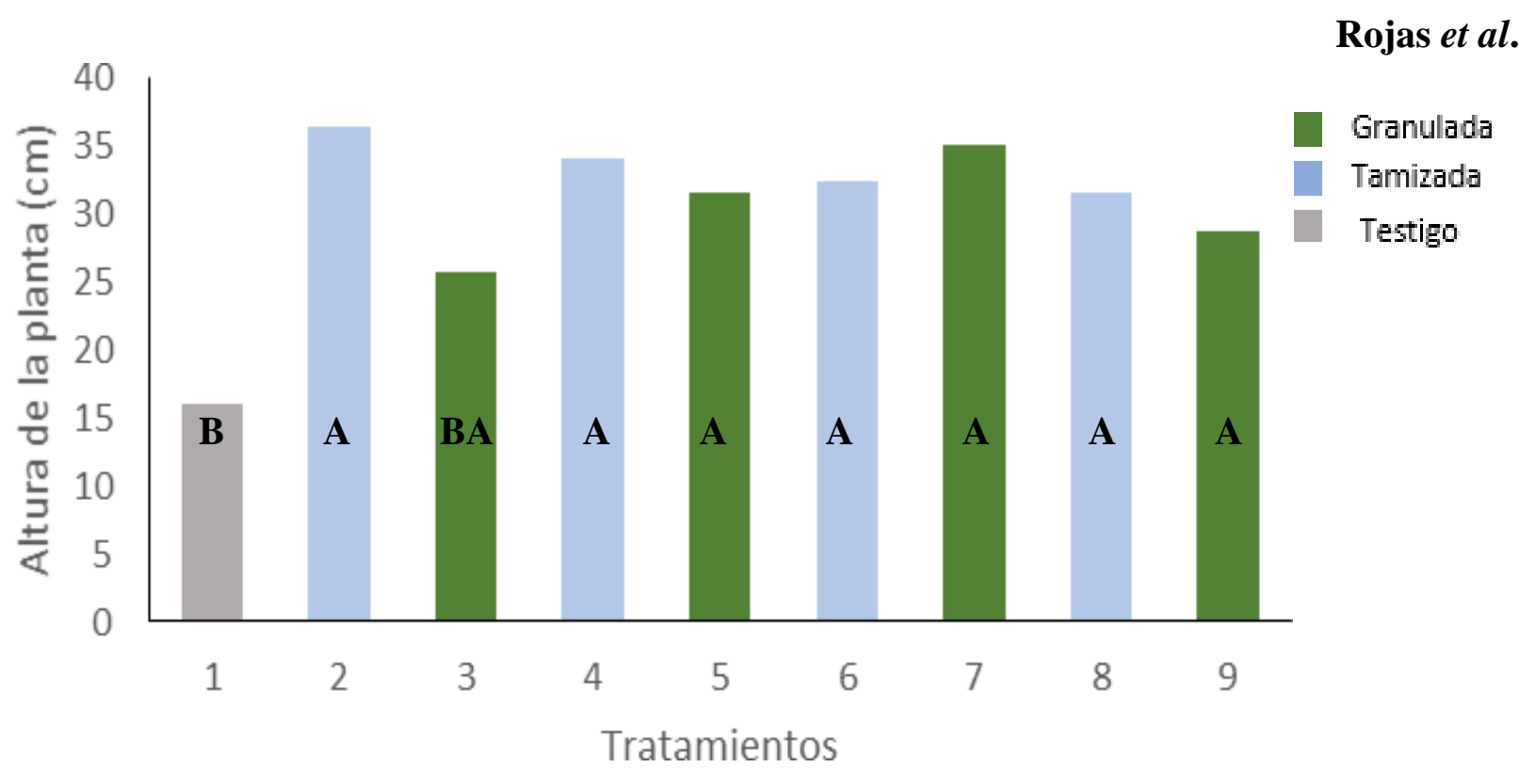

Figura 2. Gráfico de medias de altura de planta de chile habanero variedad Jaguar (Capsicum chinense $J A C Q)$. Y agrupación por prueba de Duncan.

\section{Frutos por planta}

Al realizar al análisis se encontraron diferencias significativas para tratamientos y repeticiones. En la cosecha se obtuvo un promedio de 3 a 11 frutos por planta, lo que fue comparado con lo mencionado por Muños (2008) quien menciona que en promedio una planta de chile habanero produce de 3 a 8 frutos.

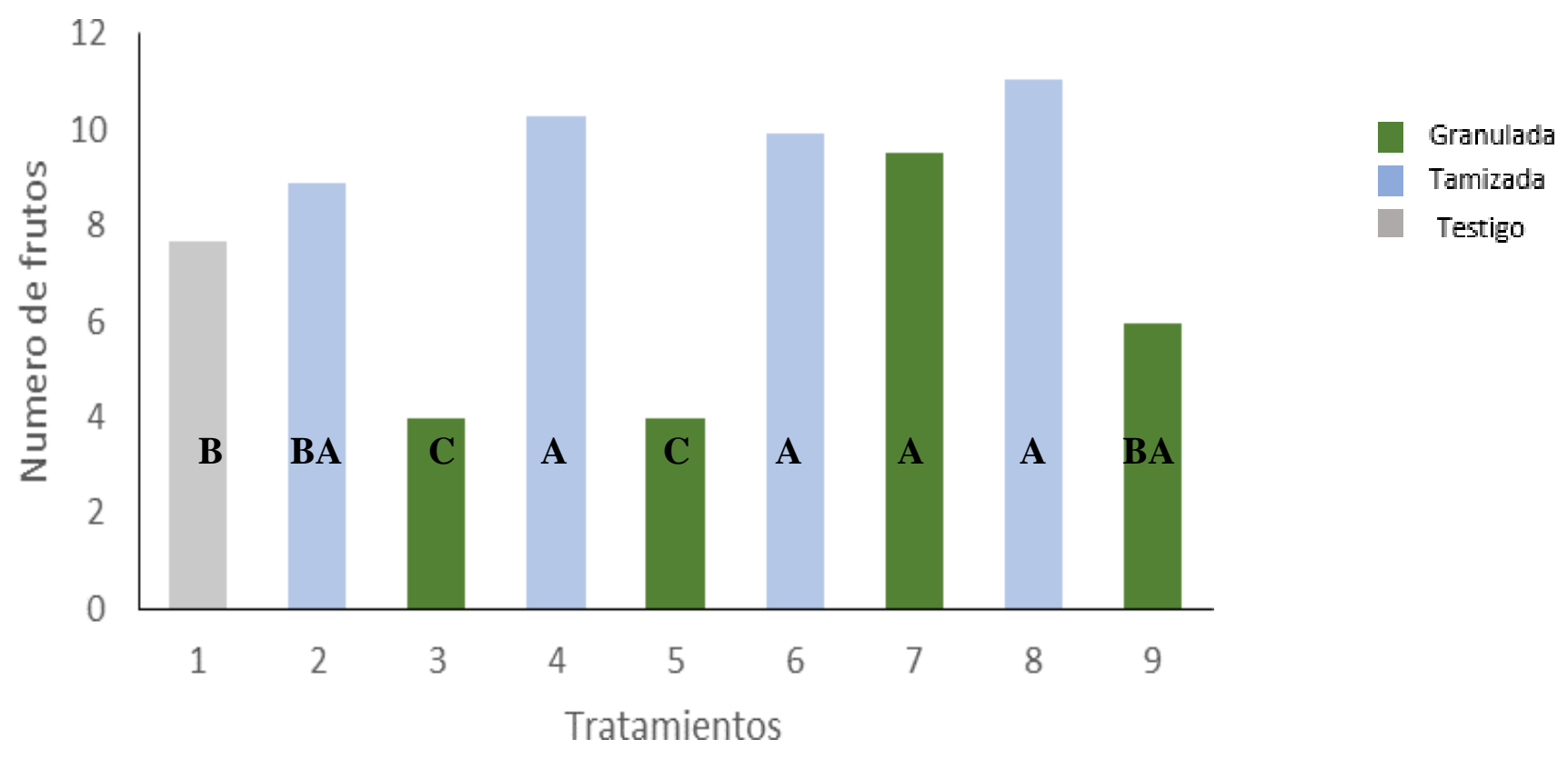

Figura 3. Gráfico de medias de número de frutos por planta de chile habanero variedad Jaguar (Capsicum chinense JACQ). Y agrupación por prueba de Duncan. 


\section{Longitud de los frutos}

De acuerdo al análisis de varianza para la longitud del fruto, se observó que existe una diferencia significativa entro los tratamientos y las repeticiones. Muños (2008) menciona que la longitud promedio de los frutos del chile habanero es de $1 \mathrm{a} 6 \mathrm{~cm}$ y en este trabajo el
Rojas et al., 2020 promedio de tamaño del fruto está dentro de la categoría con $3 \mathrm{~cm}$. De acuerdo a la agrupación de medias por Duncan observamos que el tratamiento número cinto obtuvo un mayor tamaño de frutos no obstante es estadísticamente igual a los tratamientos cuatro y ocho mientras que el tratamiento uno es estadísticamente inferior a dichos tratamientos.

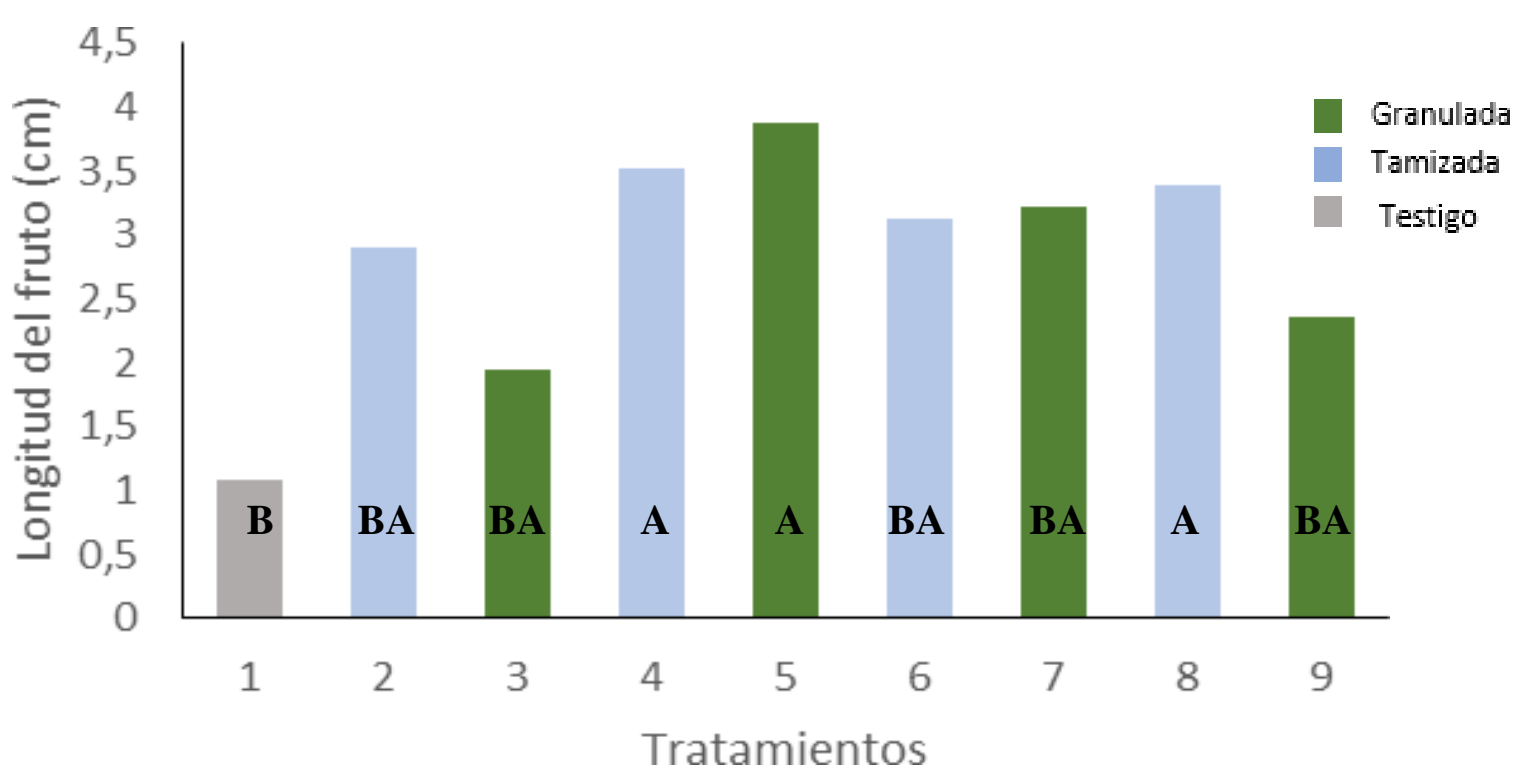

Figura 4. Gráfico de medias de longitud del fruto de chile habanero variedad Jaguar (Capsicum chinense $J A C Q)$. Y agrupación por prueba de Duncan.

\section{Diámetro del fruto}

De acuerdo con el análisis de varianza la variable diámetro de frutos, se obtuvo que existe una diferencia significativa entre los tratamientos. Con base a la prueba de Duncan se observó que el tratamiento tres es estadísticamente superior a los tratamientos tres, nueve y uno pero estadísticamente igual a los tratamientos dos, ocho, siete, cinco y cuatro. En cuanto al tratamiento uno demostró ser estadísticamente inferior. Villa (2014) menciona que el promedio para el diámetro de los frutos es de 2.7 a $4.5 \mathrm{~cm}$, por lo cual para este experimento se obtuvo un promedio de entre 1 a $3 \mathrm{~cm}$. 


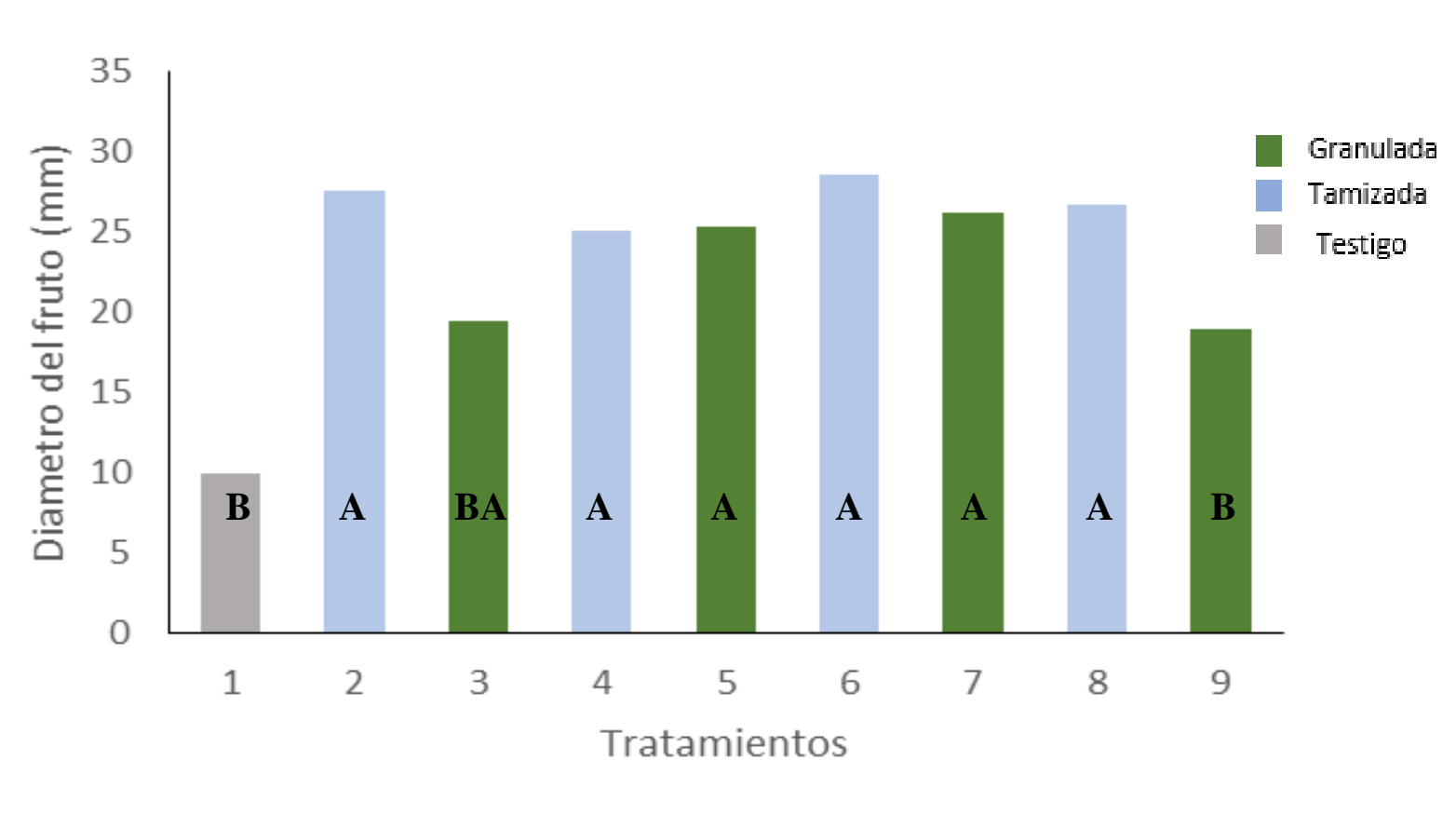

Figura 5. Gráfico de medias del diámetro del fruto de chile habanero variedad Jaguar (Capsicum chinense $J A C Q)$. Y agrupación por prueba de Duncan.

\section{Rendimiento}

De acuerdo al análisis de varianza se concluye que existe una diferencia significativa entre los tratamientos y repeticiones. Hernández (2016) menciona que el rendimiento por hectárea de chile habanero oscila entre la 5 y11 toneladas, mientras que en este trabajo de investigación el mayor rendimiento obtenido tiene en promedio una tonelada por hectárea. Con base a la prueba de medias por Duncan se observa que estadísticamente el tratamiento seis fue superior a los tratamientos nueve, cuatro, uno, siete, tres, dos y cinco. Siendo el tratamiento cinco inferior estadísticamente a los demás tratamientos. 


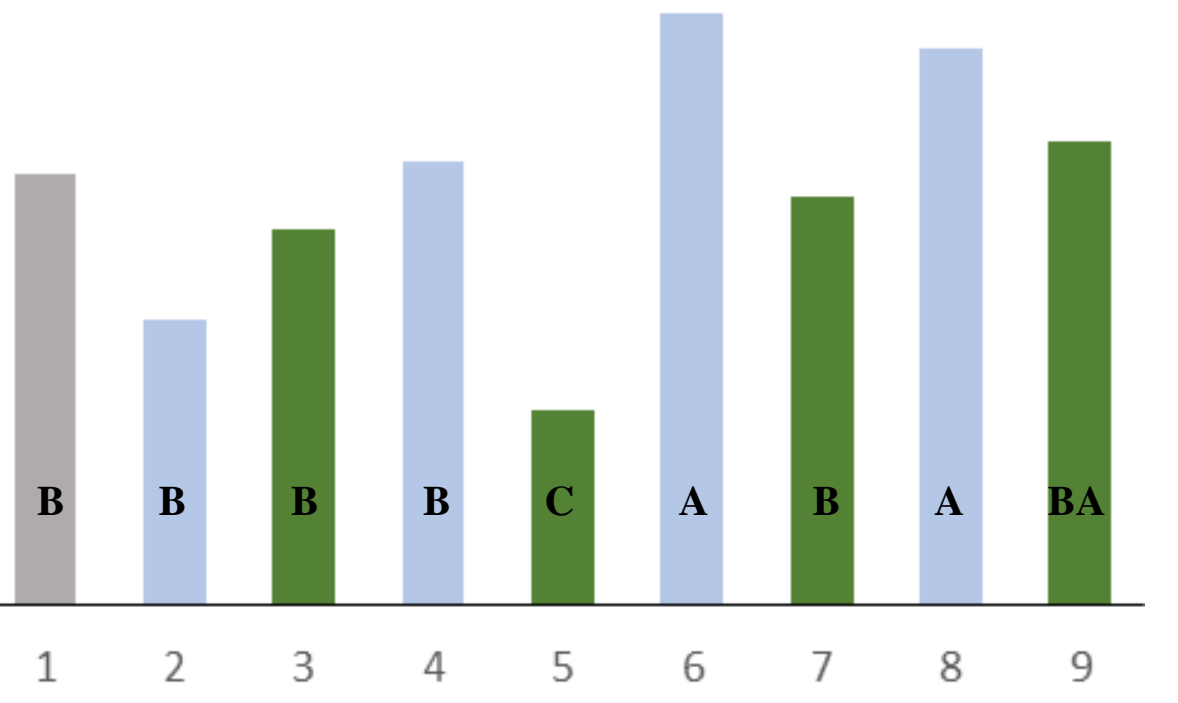

Granulada

Tamizada

Testigo

\section{Tratamientos}

Figura 6. Gráfico de medias del rendimiento promedio de chile habanero variedad Jaguar (Capsicum chinense JACQ). Y agrupación por prueba de Duncan.

\section{CONCLUSIÓN}

De acuerdo a los resultados de análisis de varianza de cada una de las variables que se evaluaron podemos decir que existe una diferencia significativa para las variables: altura de la planta, número de frutos por planta, longitud y diámetro del fruto y rendimiento en kilogramos por hectárea. Cabe resaltar que el biofertilizante tuvo un mejor efecto en la lombricomposta sin tamizar.

A pesar del escaso manejo de plagas y enfermedades, el biofertilizante Consorcio tuvo un efecto positivo en el desarrollo del cultivo de chile habanero variedad jaguar.

Debe considerarse un mejor manejo de plagas $y$ enfermedades en futuros estudios.
No se pudo comprobar la hipótesis debido a que solo se realizaron 4 cortes en este experimento por causa de la falta de abastecimiento de agua, las condiciones climáticas externas que se presentaron y la presencia de plagas en el cultivo.

\section{RECOMENDACIONES}

Se recomienda repetir el experimento evaluándolo a diferentes dosis en lombricomposta granulada o sin tamizar.

Tener en óptimas condiciones la infraestructura del invernadero para que las condiciones externas no afecten el cultivo.

Realizar un trabajo en donde se maneje más eficientemente la incidencia de plagas y enfermedades para poder realizar el análisis a un mayor número de cosechas. 


\section{LITERATURA CITADA}

ASERCA. (2014). Agencia de Servicios a la Comercialización y Desarrollo de Mercados Agropecuarios. Recuperado el 6 de 09 de 2019, de Reporte diario de diversos precios observados de diversas centrales de abasto: http://www.infoaserca.gob.mx/hortalizas nacional/hna_ca2.asp. https://doi.org/10.1787/leo-2015-graph7-es

Hernández B. R. Mateos, F. Orgaz., E. Ferrer. (2009). Potencial productivo y limítate para la producción de chile habanero (Capsicum chinense Jacq) en la zona centro del estado de Veracruz. Tesis Doctoral, Campus Veracruz. Colegio de Postgraduados, Manlio Fabio Altamirano, Veracruz. https://doi.org/10.5154/r.rchsh.2016.02.004

Chavéz D. J. (2010). Elaboración Artesanal de dos abonos líquidos fermentados y su efectividad en la producción de la plántula de chile Habanero (capsicum Chinense Jacq). Tesis Maestro en Ciencias. 41 Campus Tabasco. Colegio de Postgraduados, Cárdenas, Tabasco. https://doi.org/10.20937/rica.2019.35.02.17

Muños T. F. J. (2008). Evaluación de dos métodos de aplicación de $\mathrm{Co} 2$ en la producción intensiva de chile habanero (Capsicum chínense) bajo condiciones de invernadero. Tesis para obtener el título https://doi.org/10.18633/biotecnia.v19i1.365
Rojas et al., 2020 de Ing. Agrónomo Facultad de Ciencias Biológicas y Agropecuarias Peñuela Municipio de Amatlán de los Reyes.

SAGARPA. (2012). Recuperado el 06 de 09 de 2019, de México, potencia productora de chile: http://www.sagarpa.gob.mx/Delegacione s/yucatan/Boletines/Paginas/201208B0 58.aspx

SIAP. (2013). Recuperado el 6 de 09 de 2019, de México es primer lugar mundial de chile verde: http://www.siap.gob.mx/produccionchile-verde/https://doi.org/10.33937/reveco.2014.50

SIAP. (2014). Recuperado el 12 de 10 de 2019, de México es primer lugar mundial de chile verde: http://www.siap.gob.mx/produccionchile-verde/

Villa J. F. (2014). Fitotecnia: Bases y Tecnología de la producción agrícola. Ed. Mundi-Prensa. España. S.A. de C.V.

Vega C. R. (2013). Guía técnica computarizada de plagas y enfermedades del cultivo del chile. (Capsicum annuиm L.). Tesis profesional para obtener el título de Ing. Agrónomo, Facultad de Ciencias Biológicas y Agropecuarias Universidad Veracruzana, Xalapa - Enríquez, Ver. https://doi.org/10.29018/978-9942-823-04-5 
Copyright (c) 2020 Rojas Avelizapa Luz Irene, Dávila Lezama Maria del Rosario, Ang el Lara Maria Alva, Navarro Rodriguez Ana Mari a del Pilar, Regalado Infante Paul Edg ardo

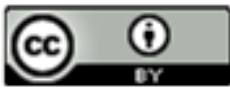

Este texto está protegido por una licencia Creative Commorr 4.0

Usted es libre para Compartir —copiar y redistribuir el material en cualquier medio o formato- y Ad aptar el documento —remezclar, transformar y crear a partir del material- para cualquier propósito, incluso para fines comerciales, siempre que cumpla la condición de:

Atribución: Usted debe dar crédito a la obra original de manera adecuada, proporcionar un enlace a la licencia, e indicar si se han realizado cambios. Puede hacerlo en cualquier forma razonable, pero no de forma tal que sugiera que tiene el apoyo del licenciante olo recibe por el usoque hace de la obra.

Resumendelicencia - Textocompletodelalicencia 